\title{
Fight or Flight
}

\author{
Designer: Rebecca Nelson, Iowa State University, USA \\ Mentor: Ling Zhang, Iowa State University, USA
}

Keywords: Handbag, Experimental, Resin, Conceptual Design

\section{Mentor Statement}

This design was a project of an undergraduate independent study. The purpose of this mentorship relationship was to help the student understand the creative design process and strategies on accessory designs to address significant social issues. As a design mentor, I gave the student advice on the creation of the handbag silhouette, and provided the resources for making the resin handles. We had a lot of discussions on design concept and visual impact. This submitted handbag embodies deep thoughts regarding mental health awareness in addition to its unique and gorgeous components, high quality of the construction, and what's more the tremendous development of the student's problem solving skills.

\section{Design Statement}

Fight or Flight is a conceptual handbag accessory with the purpose of promoting both suicide and mental health awareness in a remarkable and unique way. The fight-or-flight response, also known as acute stress response, is a physiological reaction that occurs during a perceived harmful event or threat to survival. Essentially, the response prepares the body to either fight or flee the situation ("Fight-or- flight reaction", n.d.). This concept elutes to a story of someone being the perceived threat to themselves. Someone whose life is at stake with a simple decision of fight or flee standing in the way.

The ultimate goal of this design was to use aesthetic properties to evoke emotion in the wearer as well as start a conversation in regard to mental health. Several visual properties were used to create such an impact including the embossed suicide awareness hotline number located at the bottom of the center front panel ("Lifeline", n.d.). Each number was scorn into the vinyl material by using an embossing kit and a hammer previous to placing the lining inside of the handbag (see Figure 1). Another unique and impactful aesthetic property of this design is the outline of faces on each color blocked panel. The "face" is representative of beauty, elegance and expression as well as the personality of an individual. This visual element represents the individuality of each person - especially those managing a mental illness.

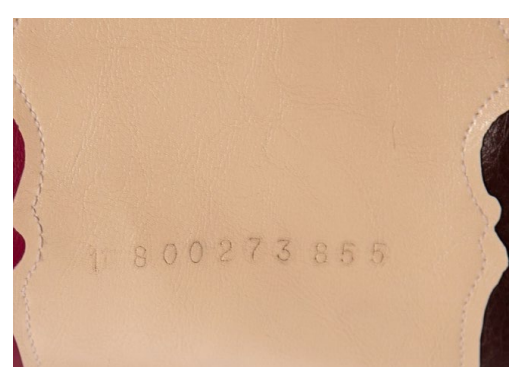

Figure 1. Embossed Leather with suicide hotline phone number.

Page 1 of 3

(c) 2020 The author(s). Published under a Creative Commons Attribution License (https://creativecommons.org/licenses/by/4.0/), which permits unrestricted use, distribution, and reproduction in any medium, provided the original work is properly cited.

ITAA Proceedings, \#77 - https://itaaonline.org 
The handles of the piece are equally as impactful. The inspiration behind this element was to portray life, frozen in time. This aspect was executed by using real, live flowers and solidifying them in resin - literally freezing them in time. While conducting trend research for S/S 2021 via WGSN, one of the most exciting forecasted trends happened to be the use of new surfaces and solid materials within fashion, particularly with accessories. "Translucent Nature" is a forecasted concept incorporating natural elements into synthetic materials to create unique designs with sentimental appeal. Each of the flowers used (delphiniums, lily's, and roses) have a specific meaning. Lily's represent the soul of the departed, roses simulate enduring passion, and delphiniums convey a feeling of lightness, levity, and an open heart. The flowers within the handles breathe life back into this design and distract the purpose from highlighting the darkness, and instead telling a love story for anyone who is contemplating a life altering decision.

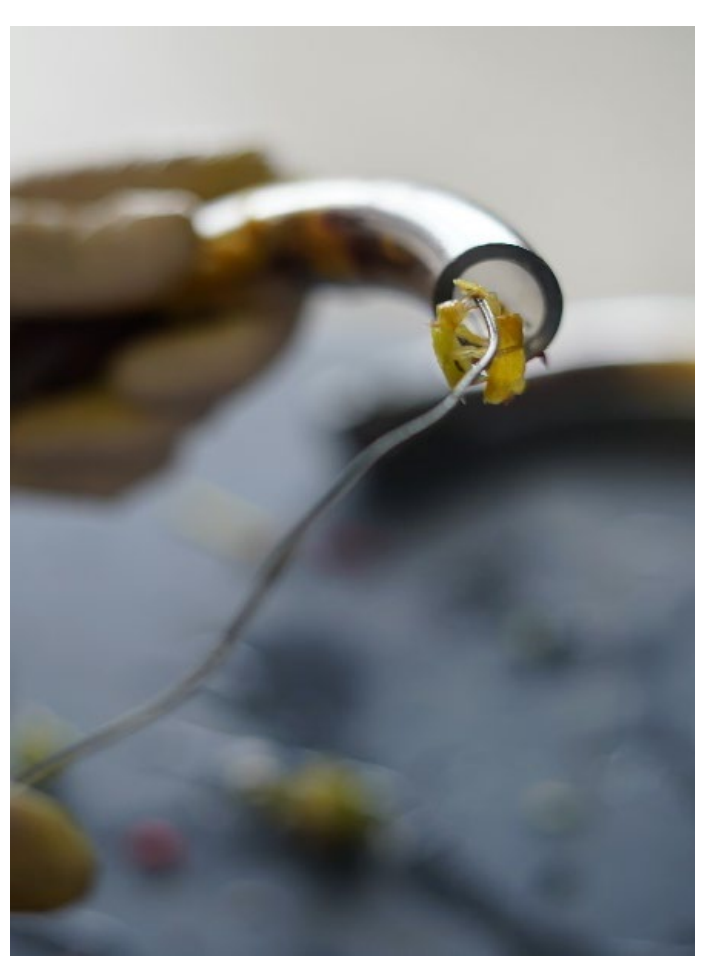

Figure 2. Placing

flowers into PVC tubing

Once the design was fully conceptualized, process techniques and execution could begin. The outer shell of the handbag was flat patterned into 9 different pieces; one bottom piece, two side panels, two centerfront panels with free-drawn faces looking away from one another, and 4 side-front panels (two red and two pink). The pieces were attached using a leather machine which offered stronger thread and a presser foot that could move across the vinyl without snagging. In order to keep the face silhouettes recognizable, top stitching the front panels together was required. To stitch along the twists and turns of the face I mainly hand spun the needle knob on the side of the machine in order to assure accuracy and precision.

The next step in executing the design was to create the resin handles. After much consideration, it was decided to use PVC tubing, generally used for plumbing purposes, as the outer vessel in which the flowers would be pushed into, and the resin would be poured through. To begin the process, the flowers were pushed through the tubing using a small wire, slowly finagling them into the proper places (see

Figure2). Once the flowers were placed throughout the piping one of the ends of the tubing was duct tape so that the resin could not pour out. After mixing the resin properly, it was slowly poured into the tubing using a funnel and sometimes pushed down using the wire, essentially swirling the flowers and the resin together (see Figure 3). Once the resin had time to settle and fully sift down to the bottom, the open end of the tubing was duct taped and then both pieces of completed tubing

Page $\mathbf{2}$ of $\mathbf{3}$ 
were placed into identical circular containers which insured that the resin would cure in the desired curved handle shape.

The resin poured handles are handcrafted, original and innovative which make them experimental, time consuming, and expensive especially when using real flowers. This would make the handbag an expensive and lucrative accessory that generally would not be easily manufactured or mass produced. In terms of the handbag itself, reproducing the design is fairly possible. Instead of using leather, different colors of vinyl were used based on availability, colors, and cost. The relationship between the different colors of vinyl show the ups, downs, twists, and turns that come our way and the choices we have and how we want to confront them. The dark red and the magenta pink are colors that would not generally be paired together however they form a unique fusion of color and make a bold statement. The cream front panels, and the steel blue lining lighten up the design and allow the colors to speak for themselves.

Fight or Flight is a visually impactful, unique, and sentimental handbag accessory that is meant to evoke an emotional conversation in regard to mental health. The combination of aesthetic and visual elements make for an interesting and complimentary piece that can elevate and add meaning to any wardrobe.

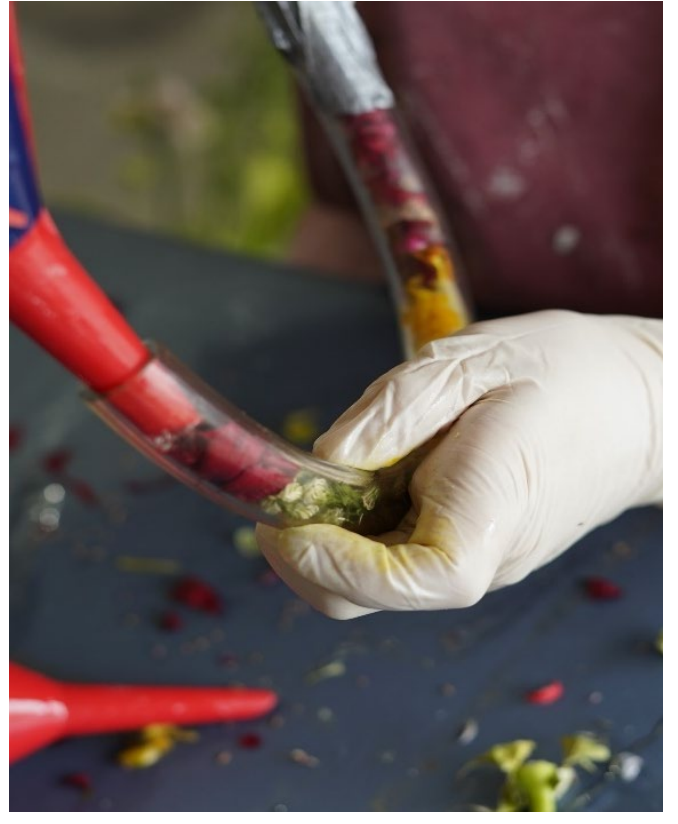

Figure 3. Pouring resin into PVC tubing

\section{References:}

Fight-or-flight reaction. (n.d.). Retrieved May 5, 2020, from https://www.dictionary.com/browse/fight-or-flight- reaction?s=ts

Lifeline. (n.d.). Retrieved May 5, 2020, from https://suicidepreventionlifeline.org/

Solid Materials Accessories, Footwear \& Jewellery. (2020). Retrieved May 5, 2020, from https://www.wgsn.com/fashion 


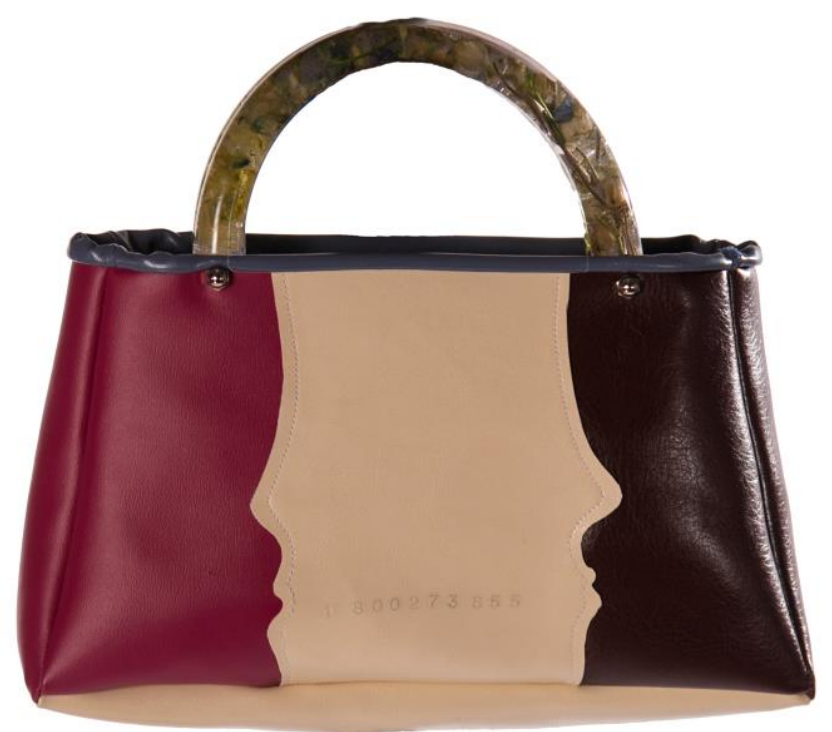

Image A: Full Front View

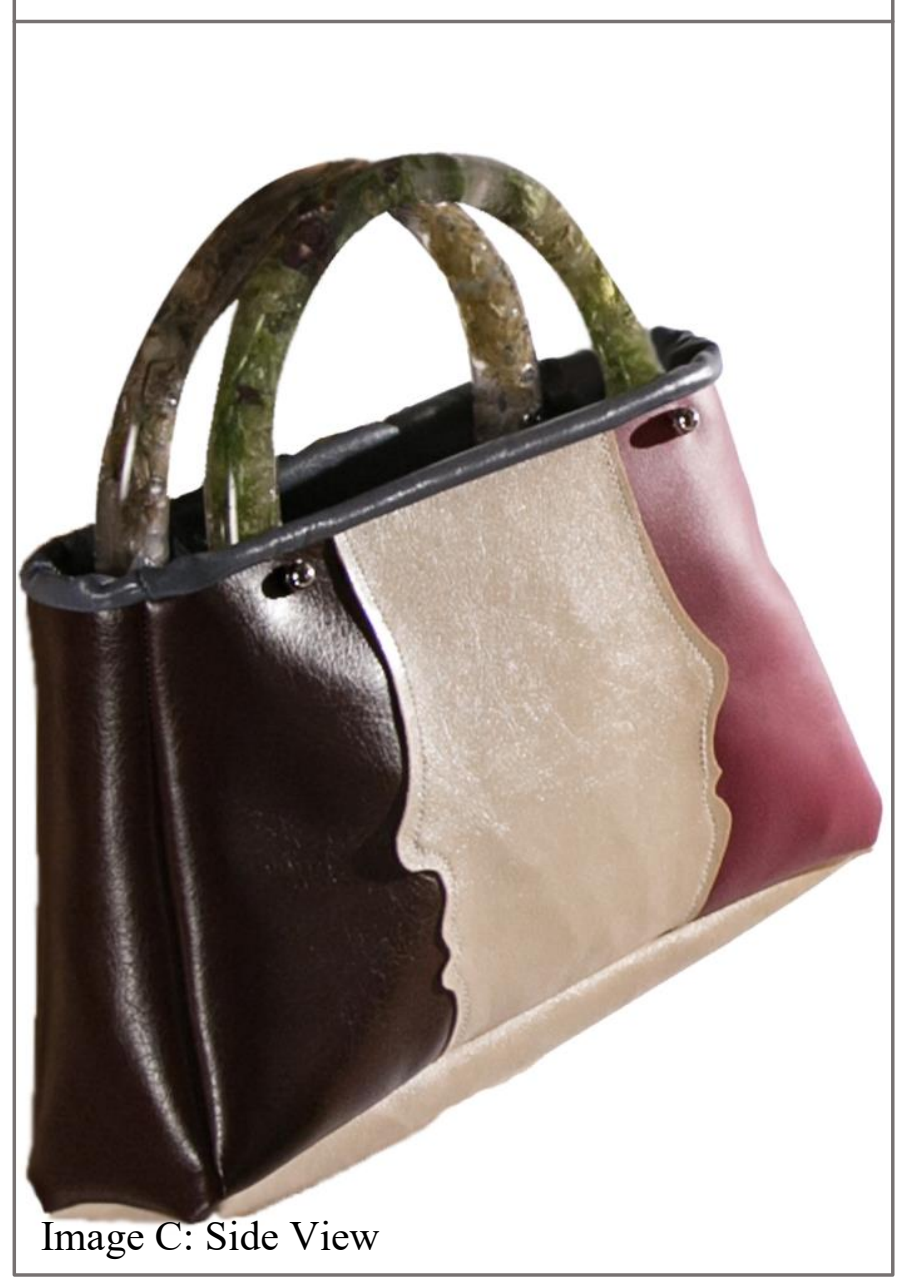

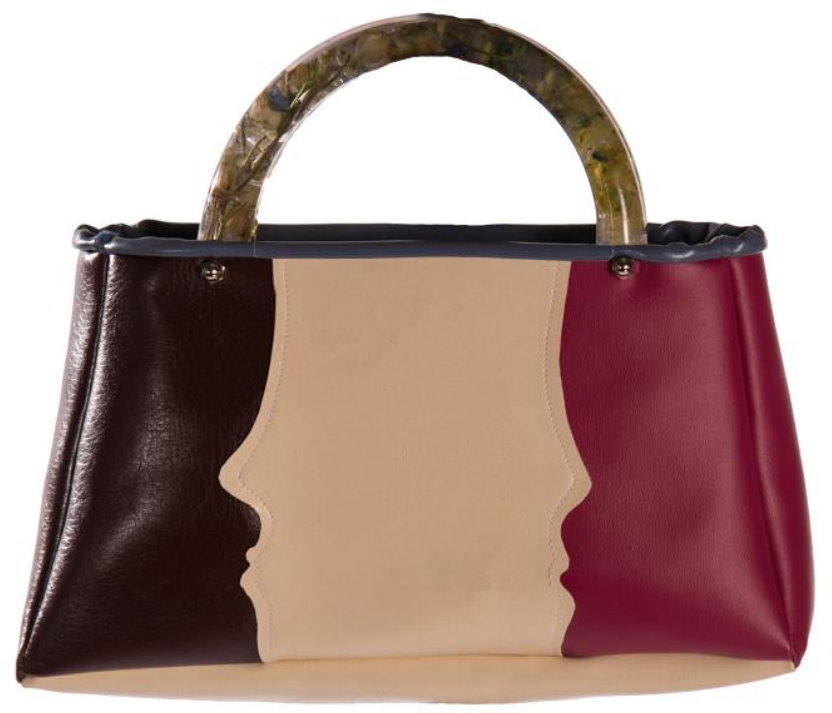

Image B: Full Back View

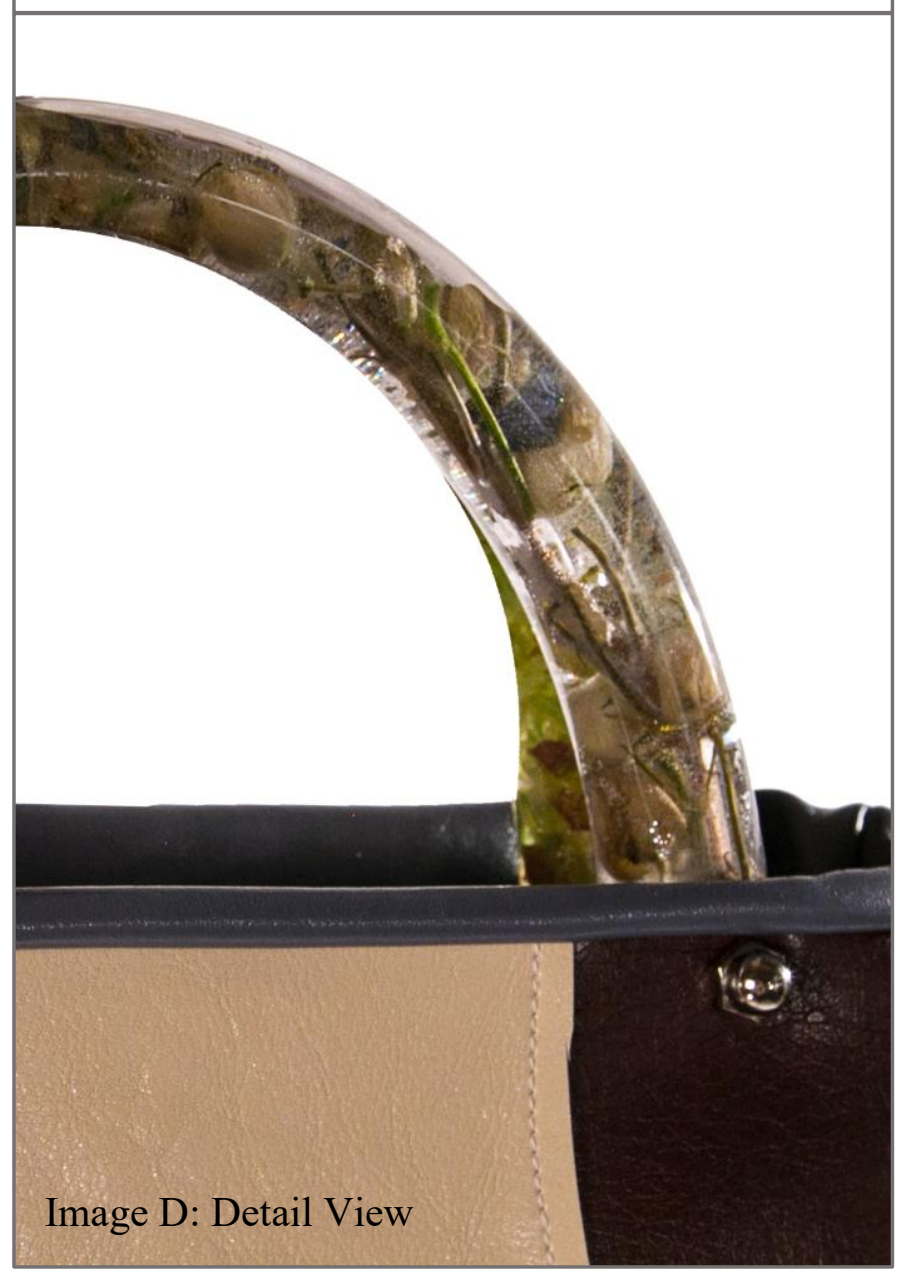

